\title{
Constrained Multi-Objective Optimization of a Common-Mode Suppression Filter
}

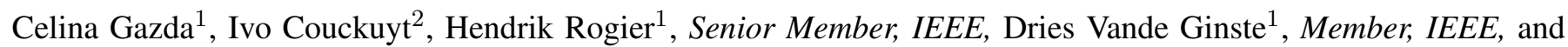 \\ Tom Dhaene ${ }^{2}$, Senior Member, IEEE
}

\begin{abstract}
Designing an optimized common-mode suppression filter for a bend in a differential trace pair on a printed circuit board involves determining the geometrical parameters that simultaneously provide a large reduction of conversion noise, a small differential mode reflection coefficient and low overall loss, given hardware and manufacturing constraints. Therefore, a novel constrained multi-objective optimization technique is proposed that relies on intermediate surrogate models of the different cost functions instead of numerically-expensive fullwave simulations, saving CPU-time and memory resources. As a result a 3D Pareto-front is created and then constrained based on hardware limitations, depicting the trade-off between the costs and allowing an easy selection of the most optimal layout geometry.
\end{abstract}

Index Terms-common-mode filter, differential signaling, Pareto-front, multi-objective optimization.

\section{INTRODUCTION}

$\mathbf{N}$ OWADAYS, many high-speed digital circuits employ differential signaling transmitted over coupled microstrip lines, given its high electromagnetic (EM) immunity against radiated and conducted noise, low EM interference and crosstalk. However, constant miniaturization of digital circuits, increasing bit rates and small noise margins require very low differential-to-common mode conversion, as this causes severe signal integrity degradation [1] and high noise levels at the receiving end that can even damage the receiver. Unfortunately, the mode conversion phenomenon easily occurs at a bend discontinuity [2].

The new structure described in [2], [3] is one of many possible filter designs that can be applied in the area of the bend to minimize mode conversion. However, although in [2] a very good suppression of common-mode noise was achieved, less attention was devoted to simultaneously minimizing reflection and losses. Therefore, in this short paper we apply a new multiobjective optimization [4] strategy to account for these, often conflicting, requirements. The novelty lies in the fact that, to speed up the process that normally relies on a large number of time-consuming full-wave simulations, we construct and exploit multiple surrogate models in a three-step approach. First, using only one limited set of full-wave simulations, six surrogate models are simultaneously constructed for the $L^{2}$ and $L^{\infty}$ norms of return loss, conversion loss and power loss. Next, a 3D Pareto-front is created by means of multi-objective

\footnotetext{
${ }^{1}$ Electromagnetics Group, Department of Information Technology, Ghen University, Sint-Pietersnieuwstraat 41, 9000 B-Gent, Belgium. E-mail: celina.gazda@intec.UGent.be, Tel.: +32 92643323

2 Department of Information Technology, Ghent University-IBBT, Gaston Crommenlaan 8, 9050 B-Gent, Belgium
}

optimization relying on the surrogate models for the $L^{2}$-norms of these three costs. Finally, the surrogate models for the $L^{\infty}$ norms are used to reduce the Pareto-optimal surface to an acceptable region satisfying the hardware constraints. Once constructed, the surrogate models allow a designer to quickly decide which design from the set of available layouts best fits the actual system requirements within the set of constraints put forward by the hardware and the manufacturing process.

In the next section we describe the filter to be optimized and its conflicting requirements, whereas Section III outlines the multi-objective optimization strategy. Section IV demonstrates how the optimal common-mode suppression filter is found by first constructing the surrogate models for the different costs, exploiting them to generate the Pareto-front, which is then restricted by a set of constraints. Finally, a set of carefully chosen samples is validated by means of full-wave simulations, to prove the accuracy and efficiency of the proposed method.

\section{COMMON-MODE SUPPRESSION FILTER}

In a pair of coupled lines, with a cross-section as in Fig. 1(a), designed on a Rogers RO4350B substrate $\left(\epsilon_{r}=3.66, \tan \delta=0.003, h=1.524 \mathrm{~mm}, t=35 \mu \mathrm{m}\right.$, and $\sigma=4.1 \cdot 10^{7} \mathrm{~S} / \mathrm{m}$, which is the value for the gold used to plate the copper microstrip lines on the board), differentialto-common mode conversion does not occur, as long as the structure remains symmetrical. However, in real printed circuit boards applications, it is impossible to avoid discontinuities such as bends. In a classic $90^{\circ}$ bend the inner line is much shorter than the outer one, causing time delay between the propagating differential signals and resulting in differential-tocommon mode conversion and unwanted noise. As a solution, we proposed to taper the coupled microstrip lines to tightly coupled ones in the area of the bend, decreasing their length difference and increasing the common-mode impedance, while the differential-mode impedance remains matched to $50 \Omega$ along the complete structure [2]. The reduction of the length difference between the two traces significantly decreases the time delay between propagating signals thereby reducing differential-to-common mode conversion. Moreover, the higher common-mode impedance and higher coupling factor between the lines in the area of the bend makes the structure act as a natural common-mode suppression filter. The verification of the applicability of the proposed structure to common mode filtering can be found in [2], which provides a validation of the reduction of common-mode noise by the new bend in the frequency-domain, and in [3], validating the new bend by means of time-domain measurements. 


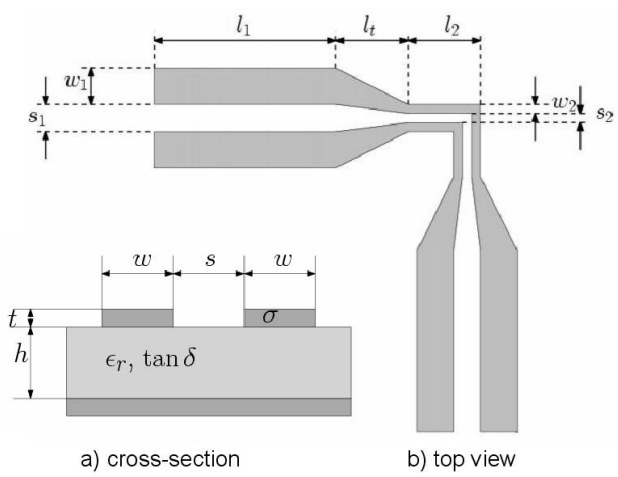

Fig. 1. Common-mode suppression filter with its geometrical parameters.

The relevant geometrical parameters for this design (Fig. 1(b)) are: cross-section of the classic coupled microstrip lines (line width $w_{1}$ and spacing $s_{1}$ ), cross-section of the tightly coupled microstrip lines $\left(w_{2}\right.$ and $\left.s_{2}\right)$, lengths $l_{1}$ and $l_{2}$ of the classic coupled microstrip lines and the tightly coupled section, respectively, and the length $l_{t}$ of the tapers. The total length of the structure is fixed to $L=l_{1}+l_{t}+l_{2}$. Unfortunately, it is not possible to find an ideal geometry providing overall optimal performance in terms of differential reflection coefficient, as well as mode conversion, overall losses, compact size, manufacturing tolerance, etc, as some of these objectives are conflicting. More tightly coupled lines in the area of the bend (smaller $w_{2}$ and $s_{2}$ ) help to reduce mode conversion but also increase total loss and reflection. Moreover, they make the structure more sensitive to production tolerances. A longer section of tightly coupled lines (larger $l_{2}$ ) prevents the tapers from directly coupling with each other but again increases the overall losses. Since the perfect solution does not exist, we need to select the most important objectives and perform a multi-objective optimization to find the set of optimal structures.

\section{Multi-objective optimization}

Popular multi-objective optimization methods rely on evolutionary algorithms. Unfortunately, those algorithms need a lot of samples to operate, which may be prohibitively expensive in the case of complex and CPU-time intensive simulation codes. A common technique for reducing the total simulation time reduces the number of required full-wave model evaluations by relying on surrogate modeling. Global surrogate modeling [5] and Multi-Objective Surrogate-Based Optimization (MOSBO) [6], [7] refer to the idea of using surrogates for the objectives in order to expedite the optimization process. A surrogate is a cheap approximation of a complex simulation model that can be used to replace that simulation model, allowing to quickly obtain any number of additional function evaluations without resorting to more expensive numerical models. The surrogate models are used to create a globally accurate approximation of each cost function in the optimization process. Subsequently, the multi-objective optimization algorithm is applied using the surrogates instead of the expensive simulation code. Although there is an initial extra cost in constructing the surrogate models, each full-wave simulation can be reused in the simultaneous construction of all cost functions required to generate a very dense Pareto-front of optimal solutions and to subsequently evaluate additional constraints imposed by hardware.

\section{Constrained Pareto Optimization of the Filter}

To determine the optimal common-mode suppression filter, e.g. in the frequency band from DC to $6 \mathrm{GHz}$, we simultaneously minimize the $L^{p}$-norms [8] of the differential reflection coefficient, the total differential-to-common mode conversion and the total losses, respectively:

$$
\begin{aligned}
\text { cost }_{1}= & {\left[\int_{0 \mathrm{GHz}}^{6 \mathrm{GHz}}\left|s_{d d 11}(f)\right|^{p} d f\right]^{1 / p} } \\
\text { cost }_{2}= & {\left[\int_{0 \mathrm{GHz}}^{6 \mathrm{GHz}}\left(\left|s_{c d 11}(f)\right|^{2}+\left|s_{c d 21}(f)\right|^{2}\right)^{p / 2} d f\right]^{1 / p} } \\
\text { cost }_{3}= & {\left[\int_{0 \mathrm{GHz}}^{6 \mathrm{GHz}}\left(P_{\text {loss }}(f)\right)^{p / 2} d f\right]^{1 / p} } \\
= & {\left[\int _ { 0 \mathrm { GHz } } ^ { 6 \mathrm { GHz } } \left(1-\left|s_{d d 11}(f)\right|^{2}-\left|s_{d d 21}(f)\right|^{2}\right.\right.} \\
& \left.\left.\quad-\left|s_{c d 11}(f)\right|^{2}-\left|s_{c d 21}(f)\right|^{2}\right)^{p / 2} d f\right]^{1 / p}
\end{aligned}
$$

where the quantities $s_{d d 11}, s_{d d 21}, s_{c d 11}$ and $s_{c d 21}$ indicate the pertinent frequency-dependent modal scattering parameters. The returned costs based on the $L^{2}$-norm correspond to the mean-squared frequency averages of the mentioned quantities, whereas the costs using the $L^{\infty}$-norms yield their maximum values (called essential supremum, [8], eq. 1.7.4).

Therefore, first, six surrogate models are constructed using least-squares support vector machines technique with lolavoronoi sampling algorithm, relying on a single set of 100 full-wave simulations: three models compute costs (1)-(3) using the $L^{2}$-norm and three models are obtained for (1)(3) based on the $L^{\infty}$-norm. The number of full-wave samples is driven by the lola-voronoi sample selector [9] that adds samples until the error of the cross-validation for all models decreases below $10^{-4}$, which provides sufficient accuracy for the surrogate models to be used as a starting point in a multiobjective optimization. The full-wave results are obtained using the planar-3D full-wave simulator ADS-Momentum. The geometrical parameters $w_{2}$ (with $s_{2}$ chosen to provide a $50 \Omega$ differential-mode impedance) and $l_{2}$ were varied over $[0.1,1] \mathrm{mm}$ and $[2,8] \mathrm{mm}$, respectively. Each cost function is approximated independently using a least-squared support vector regression surrogate model [10]. The total time needed to create all models is $5 \mathrm{~h} 2$ min using a computer with a $3 \mathrm{GHz}$ Quad CPU and with 8 GB RAM. Fig. 2 presents the surrogate models for (1)-(3) based on the $L^{2}$-norm. Figs. 2(a) and 2(b) confirm that a higher differential-mode reflection but also a better common-mode suppresion are found for smaller $w_{2}$ (a more tightly coupled cross-section in the area of the bend). In Fig. 2(c) it is visible that overall losses increase for increasing $l_{2}$ and decrease with increasing $w_{2}$. 


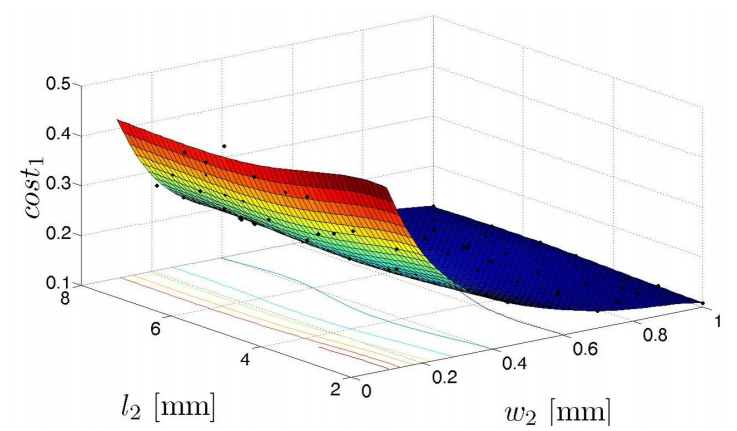

(a) Surrogate model for $\operatorname{cost}_{1}\left(L^{2}\right.$-norm).

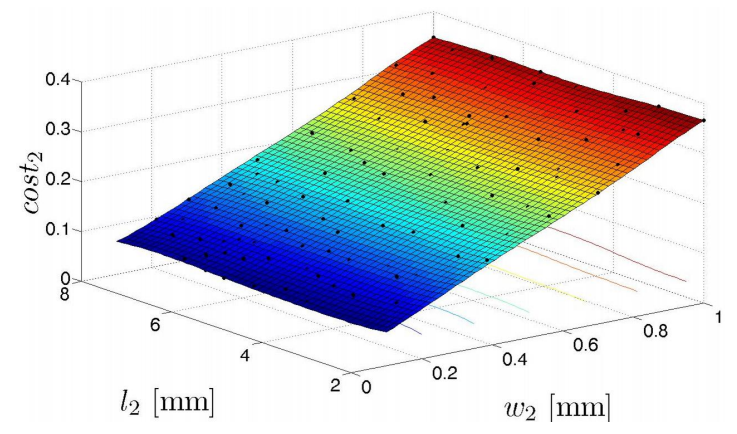

(b) Surrogate model for $\operatorname{cost}_{2}\left(L^{2}\right.$-norm).

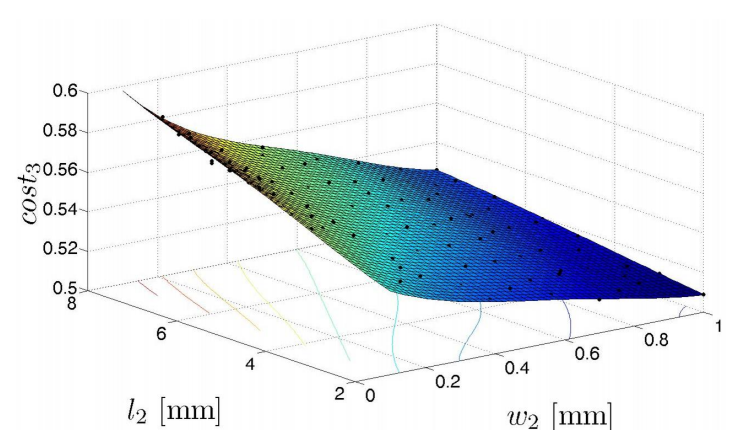

(c) Surrogate model for $\operatorname{cost}_{3}\left(L^{2}\right.$-norm).

Fig. 2. Surrogate models for the three cost functions of the chosen objectives (black dots: 100 full-wave simulation samples; surface plot: surrogate model).

Second, the multi-objective optimization is performed (taking $10 \mathrm{~min} 2 \mathrm{~s}$ using the same machine) to simultaneously minimize all three cost functions for the $L^{2}$-norms, using the SPEA2 algorithm [11] with a population size of 600 for 200 generations. If the optimization was performed based on full-wave simulations instead of relying on intermediate surrogate models, the overall simulation time would be about 25 h 12 min (600 runs, each one taking 2 min 31 s). Fig. 3(a) shows the 3D Pareto-front [12] in the performance space restricted by the ranges over which $w_{2}$ and $l_{2}$ may vary given the technology constraints. Fig. 3(b) represents the corresponding geometrical parameters of the Pareto-front samples in the design space (for clarity, in Fig. 3 only 250 samples out of 600 are shown). Designs in corner $\{1\}$ of the Paretofront, where both $w_{2}$ and $l_{2}$ are small (very short and very tightly coupled lines in the area of the bend), provide very high mode conversion suppression but also large reflection and high power losses. In corner $\{2\}$ of the front, conversion loss remains very small while the differental mode reflection decreases and the total losses increase as $l_{2}$ increases. In corner $\{3\}$, a large reduction in differential mode reflection and total losses is observed while the conversion loss increases, as the lines in the area of the bend are short and weakly coupled.

Third, additional constraints are applied to the Pareto-front to reject designs that might be harmful for the transmitter and/or receiver hardware. Using the surrogate models for the $L^{\infty}$-norms, we impose:

$$
\begin{aligned}
& \text { constraint }_{1}: \max _{f}\left|s_{d d 11}(f)\right|<-15 \mathrm{~dB} \\
& \text { constraint }_{2}: \max _{f} \sqrt{\left|s_{c d 21}(f)\right|^{2}+\left|s_{c d 11}(f)\right|^{2}}<-15 \mathrm{~dB} \\
& \text { constraint }_{3}: \max _{f} \sqrt{P_{\text {loss }}(f)}<8 \%
\end{aligned}
$$

The green circles ( $\circ$ ) on the Pareto-front represent designs satisfying all three constraints. Blue squares $(\square)$, pink leftpointing triangles $(\triangleleft)$ and dark violet triangles $(\triangle)$ indicate layouts that violate the first, second and third constraint, respectively. Orange diamonds $(\diamond)$ depict layouts violating both first and third constraint. The red star $(\star)$ indicated by the red arrow is the sample with geometry $w_{2}=0.45 \mathrm{~mm}$, $s_{2}=0.18 \mathrm{~mm}$ and $l_{2}=2.16 \mathrm{~mm}$ that provides the best solution in terms of minimal averaged insertion loss $\langle\mathrm{IL}\rangle$, obtained when all three costs are equally important, as follows: $<\mathrm{IL}>=\operatorname{cost}_{1}^{2}+\cos _{2}^{2}+\operatorname{cost}_{3}^{2}$, using the $L^{2}$-norm for the costs. This might be the designer's final choice.

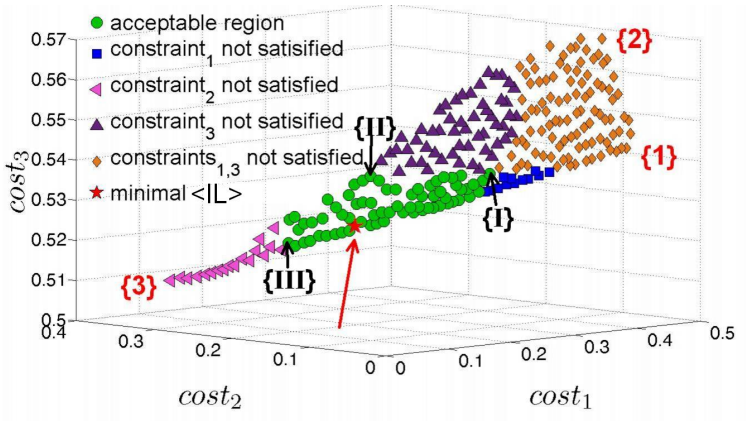

(a) 3D Pareto-front in the performance space for the three cost functions.

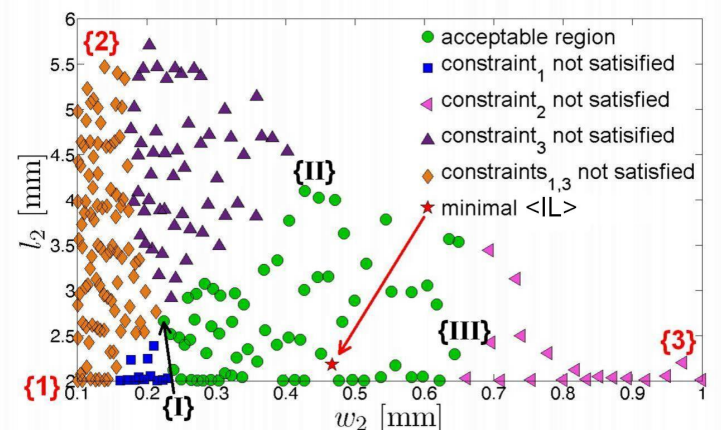

(b) Pareto samples in the design space.

Fig. 3. 3D Pareto-front and its corresponding geometrical parameters (for readibility, only 250 out of 600 samples are shown).

To validate the designs obtained by the constrained Paretofront, full-wave simulations for the best design in terms of averaged insertion loss $<\mathrm{IL}>$ marked by the red star, as well 
as for three additional layout samples (first: $w_{2}=0.22 \mathrm{~mm}$, $s_{2}=0.13, l_{2}=2.66 \mathrm{~mm}$; second: $w_{2}=0.43 \mathrm{~mm}, s_{2}=$ $0.17 \mathrm{~mm}, l_{2}=4.1 \mathrm{~mm}$; third: $w_{2}=0.64 \mathrm{~mm}, s_{2}=0.21 \mathrm{~mm}$, $l_{2}=2.3 \mathrm{~mm}$ ) were performed. These three additional samples are located in the three corners $(\{\mathrm{I}\}-\{\mathrm{III}\})$ of the acceptable (green) area, illustrating the trade-offs of the designs lying on the constrained Pareto-front. Fig. 4(a) confirms that all layouts satisfy the first constraint $\left(\right.$ cost $\left._{1}<-15 \mathrm{~dB}\right)$ and that the first green point, which is the sample laying in corner $\{\mathrm{I}\}$ of the acceptable region, is characterized by the smallest mode-conversion. Similarly, in Fig. 4(b), which shows that all layouts fulfill the second constraint $\left(\right.$ cost $\left._{2}<-15 \mathrm{~dB}\right)$; the third green point, lying in corner $\{\mathrm{III}\}$, performs the best. Fig. 4(c) proves that all selected layouts provide a very small insertion loss. Although the first green point has the smallest insertion loss at the upper frequency limit $(6 \mathrm{GHz})$, the red star point remains the best solution in terms of smallest average insertion loss $<\mathrm{IL}>$ over complete frequency range $(<\mathrm{IL}>$ equals $0.3852,0.3515,0.3494$ and 0.3428 for the first, second, third green point, and the red star, respectively).

\section{CONClusion}

A constrained multi-objective optimization strategy determining the optimal parameter set for a common-mode suppression filter has been presented. The technique uses intermediate surrogate models of the cost functions to speed up the simulation process. The set of feasable optimal designs in the 3D Pareto-front is restricted to account for hardware constraints. The presented methodology aids the designer in efficiently visualizing the design space and selecting an optimal solution. This new strategy allows EMC-aware designers to make a judicious choice about the best design given conflicting costs and while respecting the hardware limits. The application to broadband common-mode suppression filters clearly shows the practical advantages of this new design approach.

\section{REFERENCES}

[1] J. Fan, X. Ye, J. Kim, B. Archambeault, and A. Orlandi, "Signal integrity design for high-speed digital circuits: Progress and directions," IEEE Trans. on EMC, vol. 52, no. 2, pp. 392 -400, May 2010.

[2] C. Gazda, D. Vande Ginste, H. Rogier, R.-B. Wu, and D. De Zutter, "A wideband common-mode suppression filter for bend discontinuities in differential signaling using tightly coupled microstrips," IEEE Trans. Adv. Pack., vol. 33, no. 4, pp. 969-978, Nov. 2010.

[3] C. Gazda, D. Vande Ginste, H. Rogier, D. De Zutter, and R.-B. Wu, "Time domain analysis of a wideband common-mode suppression filter for bent interconnects," in 15th IEEE Workshop on Signal Propagation on Interconnects (SPI), May 2011, pp. $7-10$.

[4] Y. Li, V. Jandhyala, and H. Braunisch, "Multi-objective optimization of microprocessor package vertical interconnects," in IEEE 61st Electronic Comp. and Tech. Conf. (ECTC), Jun. 2011 2011, pp. 495-500.

[5] D. Gorissen, K. Crombecq, I. Couckuyt, P. Demeester, and T. Dhaene, "A surrogate modeling and adaptive sampling toolbox for computer based design," Journal of Machine Learning Research, vol. 11, pp. 2051-2055, Jun. 2010.

[6] G. Wang and S. Shan, "Review of metamodeling techniques in support of engineering design optimization," Journal of Mechanical Design, vol. 129, no. 4, pp. 370-380, Apr. 2007.

[7] I. Couckuyt, F. Declercq, T. Dhaene, H. Rogier, and L. Knockaert, "Surrogate-based infill optimization applied to electromagnetic problems," International Journal of RF and Microwave Computer-Aided Engineering, vol. 11, no. 5, pp. 492-501, 2010.

[8] D. Zwillinger and C. R. Company, CRC standard mathematical tables and formulae, 31st ed. CRC Press, 2003, ch. 1, pp. 72-73.

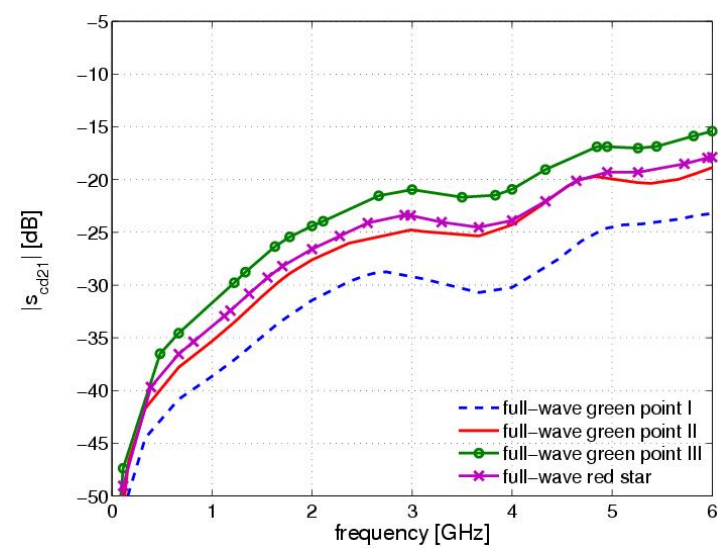

(a) Forward differential-to-common mode conversion $\left|s_{c d 21}\right|$.

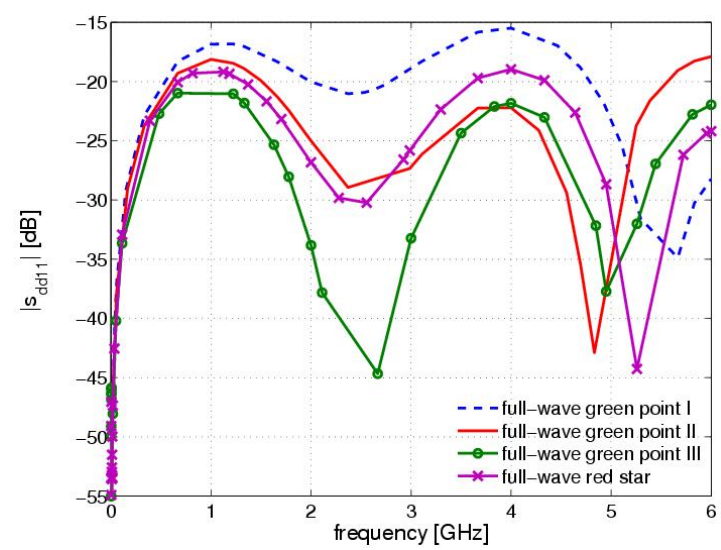

(b) Differential mode reflection coefficient $\left|s_{d d 11}\right|$.

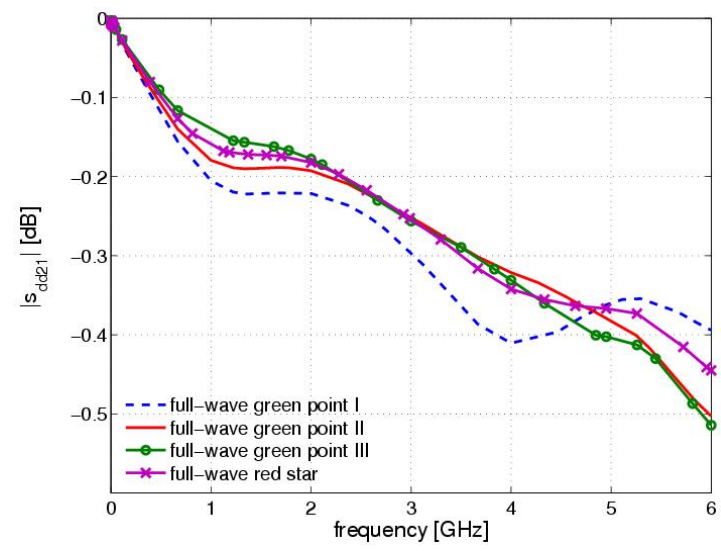

(c) Differential mode transmission coefficient $\left|s_{d d 21}\right|$.

Fig. 4. Full-wave results for the three chosen points within acceptable (green) region and for the best solution (red star).

[9] K. Crombecq, L. De Tommasi, D. Gorissen, and T. Dhaene, "A novel sequential design strategy for global surrogate modeling," in Proc. of the 2009 Winter Simulation Conference, dec. 2009, pp. $731-742$.

[10] J. de Brabanter, "LS-SVM regression modelling and its applications," Ph.D. dissertation, Katholieke Universiteit Leuven, Belgium, 2004.

[11] E. Zitzler, M. Laumanns, and L. Thiele, "SPEA2: Improving the strength pareto evolutionary algorithm for multiobjective optimization," in Evolut. Methods for Dsn Opt. and Ctrl with Appl. to Indstr. Prob. Int. Ctr for Num. Methods. in Eng., 2001, pp. 95-100.

[12] Y. Rahmat-Samii and E. Michielssen, Eds., Electromagnetic Optimization by Genetic Algorithms, 1st ed. John Wiley \& Sons, Inc., 1999, ch. 2 , pp. $48-49$. 